\title{
Detection of subarachnoid haemorrhage on early CT: is lumbar puncture still needed after a negative scan?
}

\author{
Nic van der Wee, Gabriel J E Rinkel, Djo Hasan, Jan van Gijn
}

\begin{abstract}
Computed tomography may be normal in up to $5 \%$ of patients who are investigated within one or two days after subarachnoid haemorrhage. This study investigated the need for further diagnostic evaluation after a normal CT scan was found very early (within 12 hours) in patients suspected of subarachnoid haemorrhage. A consecutive series of 175 patients with sudden headache and a normal neurological examination who had first CT within 12 hours after the onset of headache were investigated. The patients with normal CT underwent lumbar puncture, but not earlier than 12 hours after the event. Computed tomography showed subarachnoid blood in 117 patients, and was normal in 58 . Spectrophotometric analysis of CSF gave evidence for a subarachnoid haemorrhage in two of these 58 patients (3\%; $95 \%$ confidence interval (95\% CI) 0.4-12\%); a ruptured aneurysm was found in both. Thus CT was normal in two of 119 patients with a definite subarachnoid haemorrhage (2\%; $95 \%$ CI $0 \cdot 2-6 \%$ ).

It is concluded that in patients with sudden headache but normal CT a deferred lumbar puncture is necessary to rule out subarachnoid haemorrhage, even if CT is performed within 12 hours after the onset of symptoms.
\end{abstract}

$(\Im$ Neurol Neurosurg Psychiatry 1995;58:357-359)

Neurology in Utrecht,

The Netherlands

$N$ van der Wee

G J E Rinkel

$\mathrm{J}$ van Gijn

University

Department of

Neurology in

Rotterdam, The

Netherlands

D Hasan

Correspondence to: Dr Gabriel J E Rinkel, University Department of Neurolooy, Heidelbergla 100,3584 CX Utrecht The Netherlands.

Received 12 April 1994 and in revised form

16 September 1994

Accepted 27 September 1994 investigated within 12 hours and with modern CT scanners.

The period of 12 hours is important in clinical practice, as only after this delay can a lumbar puncture help in distinguishing a true haemorrhage from a traumatic tap by the presence or absence of the xanthochromic blood pigments that are formed after lysis of red cells. ${ }^{2}$

To investigate if patients with a sudden onset of headache and early but normal CT have still to be admitted for lumbar puncture after the 12 hour period has elapsed, we studied a consecutive series of patients who presented within 12 hours after the onset of sudden headache; all patients in whom no blood was found on CT underwent a deferred lumbar puncture.

\section{Patients and methods}

We studied patients admitted between January 1989 and January 1993 to the University hospitals in Utrecht and Rotterdam. Included were patients with acute headache who were oriented and had no focal deficits on examination and in whom CT was performed within 12 hours after the onset of headache. The scans were assessed for the presence of extravasated blood jointly by a neuroradiologist and at least two neurologists (including GJER or DH), all experienced in the management of patients with subarachnoid haemorrhage. Patients in whom no blood was found on CT by this panel underwent lumbar puncture, but not until at least 12 hours after the onset of headache. In patients with bloodstained CSF, or with microscopically detected erythrocytes, the supernatant fluid was spectrophotometrically examined for xanthochromia. Xanthochromia was defined as extinction exceeding 0.023 at wavelength $415 \mathrm{~nm}$ or as a peak in the absorption curve in the $450-460 \mathrm{~nm}$ range. ${ }^{2}$

All CT was performed on a Philips Tomoscan 350 or 500 (third generation scanner). Contiguous slices of $3 \mathrm{~mm}$ were used in the region of the basal cisterns. In the higher regions of the brain non-contiguous slices of $5 \mathrm{~mm}$ were made. Contrast material was not used. 
Figure 1 CT six hours after the onset of headache in a patient with $a$ ruptured posterior inferior cerebellar artery aneurysm. No blood is visible in basal cisterns or sylvian fissures.

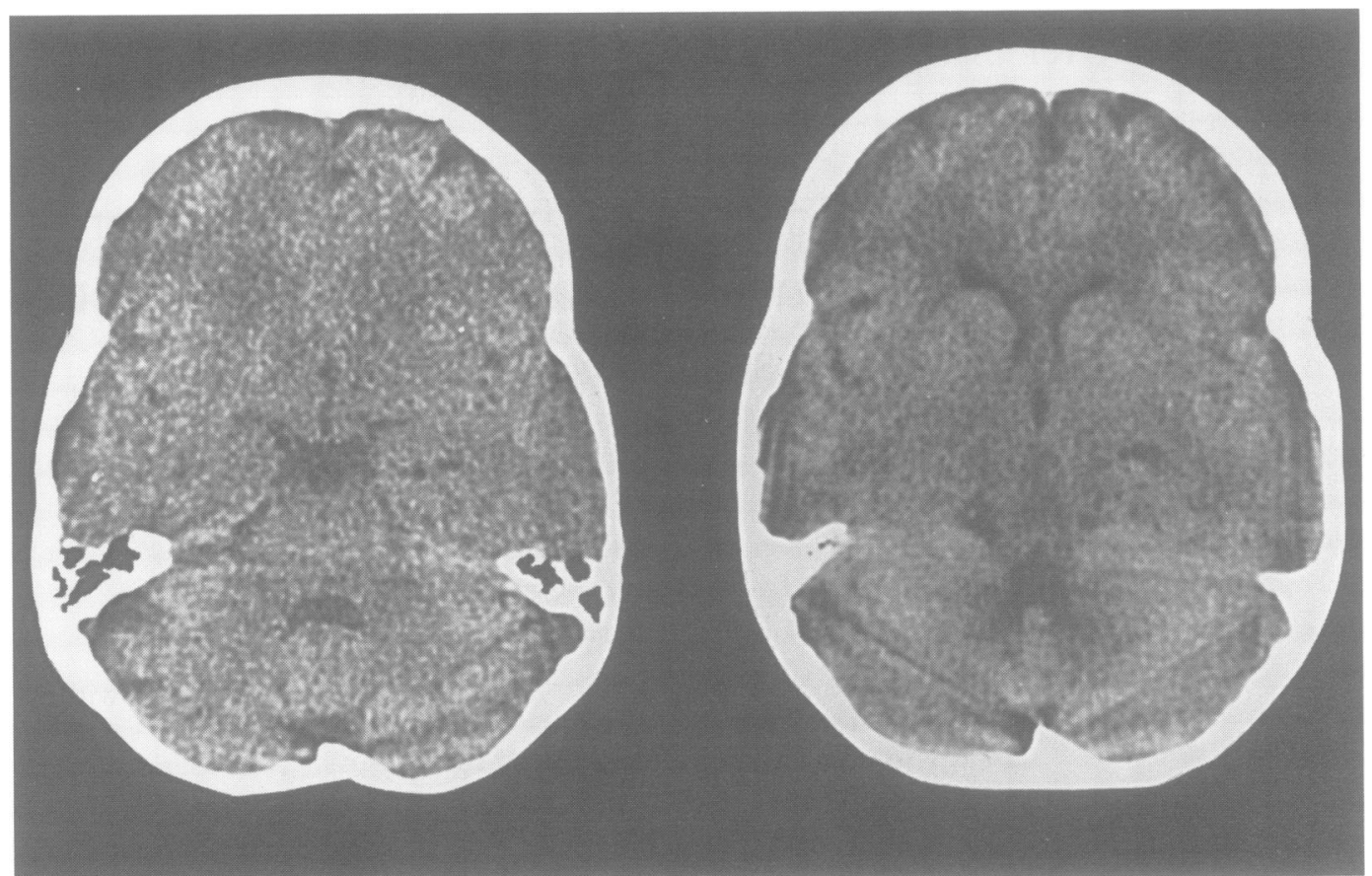

Results

During the study period 175 patients were investigated within 12 hours after the onset of headache. The panel of one neuroradiologist and at least two neurologists found evidence of blood on CT in 117 patients. The CSF was xanthochromic in two of the 58 patients with normal CT $(3 \%$; $95 \%$ confidence interval $(95 \%$ CI) $0 \cdot 4-12 \%)$. Thus CT was normal in two of 119 patients with a definite subarachnoid haemorrhage $(2 \% ; 95 \%$ CI $0 \cdot 2-6 \%)$. Re-examination of these two scans after the results of examination of CSF became known again failed to show blood or other abnormalities (fig 1).

Angiography showed an aneurysm in both patients with normal CT but xanthochromic CSF. In one of the two patients, a 50 year old woman, the aneurysm was located at the posterior inferior cerebellar artery (PICA). This aneurysm bled again during operation, but was eventually clipped, and the patient made a good recovery. The other patient, a 42 year old woman, had an aneurysm of the posterior communicating artery. Operation and postoperative course were uneventful in this patient.

In the entire series of 119 patients with proved subarachnoid haemorrhage, a ruptured aneurysm was found in 79 ; in the 117 patients with evidence of blood on CT a ruptured aneurysm was found on the anterior cerebral artery in 29 patients, on the carotid artery in 26 , on the middle cerebral artery in 17 , and on the posterior circulation in five patients. Angiography was normal in 34 patients; 22 of these patients had a nonaneurysmal perimesencephalic pattern of haemorrhage on CT. ${ }^{3}$ Six patients underwent neither angiography nor postmortem examination.

\section{Discussion}

We found that a normal CT scan does not rule out aneurysmal subarachnoid haemorrhage even in patients who present within 12 hours after the onset of acute headache and who are studied with a third generation scanner. Lumbar puncture is therefore still required in all patients with an acute headache of recent onset and normal CT; a subarachnoid haemorrhage should be suspected if CSF is bloodstained. Because spectrophotometry can reliably distinguish a true haemorrhage from a traumatic tap only if more than 12 hours have elapsed after the onset of symptoms, this implies that patients seen within that period should be admitted. The risk of a rebleed has a peak incidence within the first six hours after the initial bleed, ${ }^{4}$ and patients with a rebleed may need active life support to survive. This potential benefit for, on average, one in 30 patients warrants admission of all such patients.

In our series of 119 patients with subarachnoid haemorrhage and a normal neurological examination, the rate of detection of subarachnoid blood was $98 \%$. This is higher than in previous, prospective studies. In the Cooperative Aneurysm Study, CT was normal in $5 \%$ of all patients, including those with a depressed level of consciousness, who were investigated within one day of the haemorrhage, and in $15 \%$ of all patients who were oriented and were investigated within five days of the haemorrhage. ${ }^{1}$ The high detection rate in our study may be explained by the use of third generation CT scanners in all patients, or by the thorough scrutiny the scans received. For example, blood in the prepontine cistern is easily overlooked, and it may be the only site where subarachnoid 
Figure 2 Left: CT of a patient with a carotid artery aneurysm showing fast disappearance of extravasated blood. Upper row: CT four hours after aneurysmal rupture shows abundant blood throughout the basal cisterns. Lower row: CT 24 hours after the haemorrhage; only a smal amount of blood is left. Right: CT of a patient with a ruptured posterior communicating artery aneurysm. Four hours after the haemorrhage only a minute amount of blood is visible in the sylvian fissure.
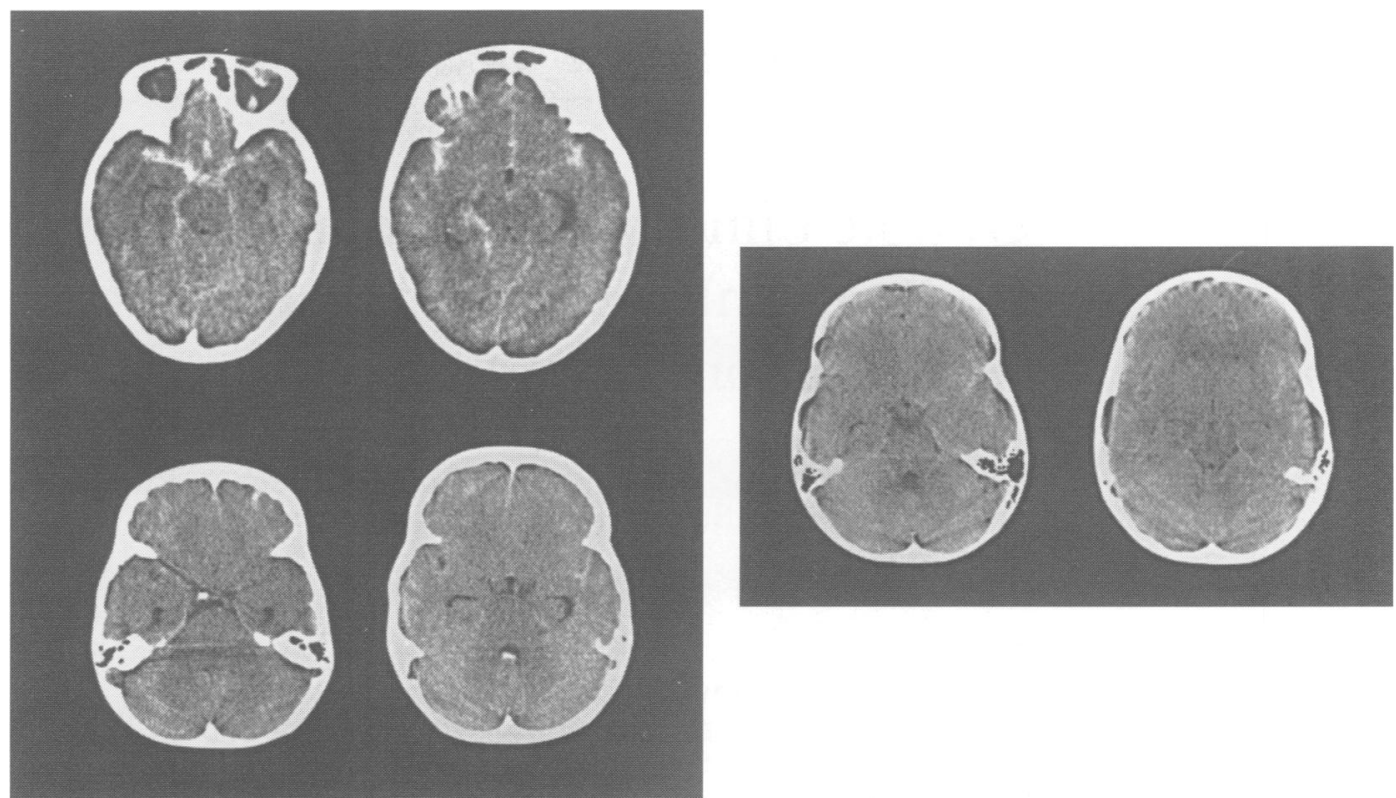

blood is found in patients with a nonaneurysmal haemorrhage. ${ }^{3}$

One of the two patients in our study with normal CT but a subarachnoid haemorrhage had an aneurysm of the PICA. Two recent reports have investigated the CT patterns of patients with posterior circulation aneurysms. Kayama and coworkers found subarachnoid blood on CT performed within three days in all 34 patients with posterior circulation aneurysms, including 12 with a PICA aneurysm. ${ }^{5}$ In another series of 20 patients with ruptured posterior circulation aneurysms, including 13 with a PICA aneurysm, subarachnoid blood was found in 13 patients and intraventricular blood in six of the seven patients in whom no blood was visible in the basal cisterns; only one patient in this series had no evidence of blood at all on CT. In this patient, who had an aneurysm at the vertebrobasilar junction, the delay between the onset of symptoms and CT was less than three days. ${ }^{6}$ Thus even in patients with a ruptured PICA aneurysm, CT is nearly always informative. The rare exceptions may be explained by the many artifacts on CT slices near the base of the skull, or by a downward directed jet of blood that prevents blood being visible in the basal cisterns. The explanations for non-detection of haemorrhage from a ruptured PICA aneurysm do not account for the second patient with nor- mal CT who had a ruptured posterior communicating artery aneurysm. Two phenomena may explain the non-detection of blood on an early CT in patients with a ruptured aneurysm on the circle of Willis. Firstly, in some patients extravasated blood disappears very quickly (fig 2 , left) and occasionally the blood may disappear too fast to be detected on CT even within 12 hours. Secondly, some patients have only a small amount of blood on an initial CT (fig 2, right) and in sporadic patients the extravasation might be too small to be detected.

We thank Drs LMP Ramos and HLJ Tanghe, neuroradiolo-

1 Adams HP Jr, Kassell NF, Torner JC, Sahs AL. CT and clinical correlations in recent aneurysmal subarachnoid Aneurysm Study. Neurology 1983;33:981-8.

2 Vermeulen $M$, van Gijn J. The diagnosis of subarachnoid haemorrhage. I Neurol Neurosurg Psychiatry 1990;53: 365-72.

3 Rinkel GJE, Wijdicks EFM, Vermeulen $M$, et al. Nonaneurysmal perimesencephalic subarachnoid hemorrhage: CT and MR patterns that differ from aneurysmal rupture. AFNR Am f Neuroradiol 1991;12:829-34;

4 Inagawa $T$, Kamiya $K$, Ogasawara H, Yano $T$. Rebleeding Surg Neurol 1987;28:93-9.

5 Kayama T, Sugawara T, Sakurai Y, Ogawa A, Onuma T, Yoshimoto T. Early CT features of ruptured cerebral aneurysms of the posterior cranial fossa. Acta Neurochir 1991;108:34-9.

6 Sadato N, Numaguchi Y, Rigamonti D, Salcman M, Gellad FE, Kishikawa T. Bleeding patterns in ruptured posterior fossa aneurysms: a CT study. $\mathcal{f}$ Comput Assist Tomogr 1991;15:612-7. gists, for their cooperation during the study. hemorrhage: a preliminary report of the Cooperative of ruptured intracranial aneurysms in the acute stage. 